\title{
Granulomatous Nodules: Know the Ropes
}

\author{
Oxana Munteanu
}

Department of Pneumology and Allergology, State University of Medicine and Pharmacy "Nicolae Testemitanu", Chisinau, Moldova

Solitary pulmonary nodules (SPNs) are commonly encountered in clinical practice, and the management involves a wide variety of dilemma, from the imaging method to be applied to the invasive approach used for the diagnosis and treatment. Aiming to improve the accuracy of SPN diagnosis, in the era of various emerging novel techniques, rational use of imaging, each one with its own strengths and pitfalls, is crucial.

The noninvasive diagnosis of pulmonary nodules remains challenging, and there is no single algorithm or "one-size-fits-all" pathway for workup of SPNs [1,2]. Outlining appropriate imaging follow-up at various time intervals, according to patient risk stratification and nodule size, the Fleischner Society guidelines for management of pulmonary nodules are widely accepted [3].

There is rising evidence that the differentiation of the malignant from benign lesions using the maximum standardized uptake value (SUVmax) threshold is unrealistic, and SUVmax of 2.5 should not be embraced as a magic threshold [4]. In geographical regions where there are high endemic rates of infectious or granulomatous lung disease (e.g., tuberculosis, nontuberculous mycobacteria [NTM], or histoplasmosis), ${ }^{18}$ F-fluorodeoxyglucose-positron emission tomography (FDG-PET)/CT may have significant limitations. A study of 279 patients in the

\section{KARGER}

(C) 2017 S. Karger AG, Basel

E-Mail karger@karger.com

www.karger.com/res
South Central United States with a high prevalence of histoplasmosis reported a specificity of $40 \%$ using FDG-PET for the diagnosis of lung cancer in pulmonary nodules [5]. In clinical practice, many pulmonary granulomas remain unexplained even after histologic examination. Since infection is the most common cause of pulmonary granulomas, it is always important to carefully exclude an infrequent etiology.

In a recent issue of Respiration, Sakakibara et al. [6] evaluated the radiopathologic features of mycobacterial infections in granulomas suspected to be lung cancers, the methods used to identify the infections, and the correlations with SUVmax.

Diagnosis of infections caused by mycobacteria, especially NTM, still represents a difficult task in both the microbiology and pathology of SPNs. Morphologically, caseating and noncaseating granulomatous reactions can occur, which often do not allow a differentiation between various infectious etiologies and hypersensitivity reactions. Regarding the Ziehl-Neelsen stain, an important point to remember is that, in most cases, mycobacteria are few and difficult to find, a fact also described by Sakakibara et al. [6] (Ziehl-Neelsen staining was positive in only 3 cases [14\%], although 16 [73\%] of the nodules had caseous necrosis). It is not uncommon to examine several

Dr. Oxana Munteanu

Department of Pneumology and Allergology State University of Medicine and Pharmacy "Nicolae Testemitanu" Stefan cel Mare si Sfant Boulevard 165, MD-2004 Chisinau (Moldova) E-Mail obarbieru@yahoo.com 
large necrotizing granulomas but find only a few mycobacteria. AFB culture was diagnostic of nearly half of the studied granuloma cases, while immunohistochemical staining had the highest sensitivity [6].

Even after a thorough review of clinical information, histologic, and bacteriological tests, 14\% of the pulmonary granulomas remained unexplained [6]. Similar cases have previously been reported by Ulbright and Katzenstein [7]. The numerous histologic similarities between such cases and those with identified organisms suggest that the former may represent „burnt-out” infections in which organisms are undetectable by currently available diagnostic tools or are eliminated by the granulomatous process.

Granulomas are dynamic lesions with cells dying continuously, debris being removed and new cells entering. While earlier studies found viable M. tuberculosis in the majority of fibrocaseous lesions in the upper areas of the lung, other studies demonstrated that up to $50 \%$ of the necrotic lesions and $85 \%$ of the calcified lesions were sterile [8]. It is accepted that the spectrum of mycobacterial infections is multifaceted, from the death of the organism to latent or active infection. Differences obtained between the cultures and immunohistochemical staining results and the association of the culture result with the nodule size in the report by Sakakibara et al. [6] highlight the fact that there is an unmet need for large studies that will allow us to better define the role and value of mycobacteria status within the granuloma.

Many pitfalls remain in the modern imaging with false-positive and false-negative nodules. Using PET-CT as a diagnostic tool could reduce the number of unnecessary biopsies or thoracotomies in benign SPNs, but PETCT cannot replace the current gold standard pathology by resection or percutaneous biopsy for diagnosing pulmonary nodules $[9,10]$. If there is no abnormality in PET images, there will be neither malignancy nor active inflammation in the lesion, and a wait-and-watch approach could be the best strategy.

It should also be emphasized that while $63 \%$ of the granulomas had a high ${ }^{18}$ F-FDG uptake, none exhibited the correlation between SUVmax and nodule size normally observed in cancer [6]. The absence of this correlation might be attributable to differences between infectious disease and cancer and could be another key consideration in the management of SPNs, but large multicenter trials are required.

\section{References}

1 Ost D, Fein AM, Feinsilver SH: Clinical practice. The solitary pulmonary nodule. N Engl J Med 2003;348:2535-2542.

2 Khouri NF, Meziane MA, Zerhouni EA, Fishman EK, Siegelman SS: The solitary pulmonary nodule. Assessment, diagnosis, and management. Chest 1987;91:128-133.

3 Macmahon H, Austin JH, Gamsu G, et al: Guidelines for management of small pulmonary nodules detected on CT scans: a statement from the Fleischner Society. Radiology 2005;237:395-400.
4 Kwee TC, Cheng G, Lam MG, et al: SUVmax of 2.5 should not be embraced as a magic threshold for separating benign from malignant lesions. Eur J Nucl Med Mol Imaging 2013;40:1475-1477.

5 Deppen S, Putnam JB Jr, Andrade G, et al: Accuracy of FDG-PET to diagnose lung cancer in a region of endemic granulomatous disease. Ann Thorac Surg 2011;92:428-432.

6 Sakakibara Y, Suzuki Y, Fujie T, Akashi T, Iida T, Miyazaki Y, Eishi Y, Inase N: Radiopathological features and identification of mycobacterial infections in granulomatous nodules resected from the lung. Respiration 2017;93:264-270.
7 Ulbright TM, Katzenstein A: Solitary necrotizing granulomas on the lung. Am J Surg Pathol 1980;4:13-28.

8 Mack U, Migliori GB, et al: LTBI: latent tuberculosis infection or lasting immune responses to M. tuberculosis? A TBNET consensus statement. Eur Respir J 2009;33:956-973.

9 Harzheim D, Eberhardt R, Hoffmann H, Herth F: The solitary pulmonary nodule. Respiration 2015;90:160-172.

10 Gould MK, Fletcher J, Iannettoni MD, et al: Evaluation of patients with pulmonary nodules: when is it lung cancer? ACCP evidencebased clinical practice guidelines (2nd edition). Chest 2007;132:108S-130S. 